\title{
Rapid In Vivo Transport and Catabolism of Human Apolipoprotein A-IV-1 and Slower Catabolism of the ApoA-IV-2 Isoprotein
}

\author{
Daniel J. Rader, Jürgen Schäfer, * Peter Lohse, Bruno Verges, Marie Kindt, Loren A. Zech, \\ Armin Steinmetz, * and H. Bryan Brewer, Jr. \\ Molecular Disease Branch, National Heart, Lung, and Blood Institute, National Institutes of Health, Bethesda, Maryland 20892; \\ and *Phillipps University Marburg Center for Internal Medicine, Division of Endocrinology and Metabolism Marburg, Germany
}

\begin{abstract}
Apolipoprotein (apo) A-IV is a polymorphic, intestinally derived apolipoprotein that is genetically linked to and similar in structure to apoA-I, the major apolipoprotein in high density lipoproteins (HDL). ApoA-IV plays a potentially important role in lipoprotein metabolism and reverse cholesterol transport, but its in vivo metabolism is poorly understood. In order to gain insight into factors modulating apoA-IV metabolism in humans, the in vivo kinetics of the two major human apoA-IV isoproteins apoA-IV-1 and apoA-IV-2 were investigated in normolipidemic human subjects. ${ }^{131} \mathrm{I}$-apoA-IV-1 and ${ }^{125} \mathrm{I}$-apoAIV-2 were reassociated with autologous plasma and injected into study subjects. Analysis of the kinetic data revealed a rapid mean fractional catabolic rate (FCR) for apoA-IV-1 of $2.42 \pm 0.11 \mathrm{~d}^{-1}$. The mean production, or transport, rate of apoA-IV-1 was $16.3 \pm 1.4 \mathrm{mg} / \mathrm{kg}$ per d. Plasma apoA-IV concentrations were highly correlated with apoA-IV production rate $(r=0.84, P<0.001)$ and not correlated with apoA-IV fractional catabolic rate $(r=0.25, P=N S)$. The mean FCR of apoA-IV-2 was $2.21 \pm 0.10 \mathrm{~d}^{-1}$. In the ten subjects in whom ${ }^{131} \mathrm{I}$-apoA-IV-1 and ${ }^{125} \mathrm{I}$-apoA-IV-2 were simultaneously injected, the FCR of apoA-IV-2 was significantly slower by paired $t$ test $(P=0.003)$. The FCR of apoA-IV-2 in an apoAIV-2 / 2 homozygote was only $1.49 \mathrm{~d}^{-1}$, substantially slower than in all other subjects. We conclude that: $(a)$ apoA-IV is a rapidly catabolized apolipoprotein in humans, with a fractional catabolic rate more than 10 times greater than that of apoA-I; (b) apoA-IV has a high absolute transport rate similar to that of apoA-I; (c) plasma levels of apoA-IV are primarily determined by apoA-IV production rate in normolipidemic subjects; and $(d)$ the fractional catabolic rate of the common variant apoA-IV-2 is slower than that of the wild-type apoA-IV-1. ( $J$. Clin. Invest. 1993. 92:1009-1017.) Key words: high density lipoproteins $\bullet$ cholesterol $\bullet$ atherosclerosis $\bullet$ kinetics $\bullet$ triglycerides
\end{abstract}

\section{Introduction}

Human apo A-IV is a $46-\mathrm{kD}$ apolipoprotein (1-5) which is synthesized primarily or exclusively in the small intestine in

Address correspondence and requests for reprints to Dr. Daniel Rader, Building 10, Room 7N117, National Institutes of Health, 9000 Rockville Pike, Bethesda, MD 20892.

Dr. Lohse's present address is University of Munich, Department of Clinical Chemistry, Munich, Germany.

Dr. Verges's present address is Hôpital du Bocage, Department of Internal Medicine, Dijon, France.

Received for publication 8 October 1992 and in revised form 18 March 1993.

The Journal of Clinical Investigation, Inc.

Volume 92, August 1993, 1009-1017 humans (6-8). The human apoA-IV gene exists as part of a gene cluster on chromosome 11 with apoA-I and apoC-III and is thought to have arisen by duplication of the apoA-I gene (9). Though its precise function remains unclear, apoA-IV has been proposed to play a role in the metabolism of both triglyceride-rich lipoproteins (TRL) ${ }^{1}$ and HDL. In addition to its presence in plasma, apoA-IV has been found in dog peripheral lymph (10) and in human mesenteric and thoracic duct lymph $(1,2)$ and interstitial fluid $(11,12)$. ApoA-IV has been shown to modulate lipoprotein lipase (LPL) activity (13), bind to endothelial cells (14), stimulate cholesterol efflux from adipose cells $(15,16)$, activate lecithin:cholesterol acyltransferase (LCAT) $(17,18)$, participate in HDL particle conversion by cholesteryl ester transfer protein (CETP) $(19,20)$, and bind specifically to hepatic tissue $(21,22)$. Weinberg et al. (23) recently demonstrated that apoA-IV exhibits labile reversible binding to $\mathrm{HDL}_{3}$ and proposed that apoA-IV helps to maintain optimal surface pressure for LCAT and CETP activities. As a result of its distribution and in vitro properties, apoA-IV is thought to play a potentially important role in reverse cholesterol transport.

ApoA-IV is a genetically polymorphic apolipoprotein in humans (24-26). The major allele, known as apoA-IV-1, has an estimated gene frequency of 0.91 , while the most common variant, apoA-IV-2, has a gene frequency of $\sim 0.08(25-30)$. We recently reported that the molecular basis for this common polymorphism is a single $\mathrm{G}$ to $\mathrm{T}$ substitution in the apoA-IV-2 allele, resulting in the conversion of a glutamine to a histidine at residue 360 of the mature protein (31). This finding has since been confirmed by two other investigators $(32,33)$. This single amino acid substitution may have an important effect on plasma lipoprotein metabolism, as heterozygotes for apoA-IV2 have been reported to have higher HDL cholesterol $(28,30)$, lower triglyceride $(30,34)$, higher LDL cholesterol $(35)$, and lower $\operatorname{Lp}(\mathrm{a})$ (35) levels than apoA-IV-1/1 homozygotes. Weinberg et al. ( 36 ) reported that the apoA-IV-2 isoprotein has more alpha-helical structure, higher affinity for phospholipids, and greater ability to activate LCAT than the apoA-IV-1 isoprotein. These structural and functional differences suggested that apoA-IV-1 and apoA-IV-2 may have different in vivo metabolism.

Kinetic studies of apoA-IV in humans have been limited to the injection of radiolabeled apoA-IV that was reassociated in vitro with ultracentrifugally isolated lipoprotein density fractions before injection into a small number of individuals, some

1. Abbreviations used in this paper: CETP, cholesteryl ester transfer protein; FCR, fractional catabolic rate; FPLC, fast performance liquid chromatography; IEF, isoelectric focusing; LCAT, lecithin:cholesterol acyltransferase; LPL, lipoprotein lipase; PR, production rate; TRL, triglyceride-rich lipoproteins. 
of whom were hyperlipidemic (37-39). Furthermore, previous kinetic studies have been performed without knowledge of the apoA-IV phenotype of the source of the purified apoA-IV or of the study subjects. In order to gain further insight into apoA-IV metabolism in humans, we performed a series of kinetic studies of apoA-IV-1 and apoA-IV-2 in normolipidemic subjects.

\section{Methods}

Study subjects. The study subjects were healthy normolipidemic subjects and included 12 apoA-IV-1 / 1 homozygotes, 7 apoA-IV-2/ 1 heterozygotes, and 1 apoA-IV-2/2 homozygote. All subjects had normal fasting glucose levels and normal thyroid, liver, and kidney function. 1 wk before the start of the kinetic study, the subjects were placed on an isoweight diet containing $37 \%$ of calories as fat $(\mathrm{P} / \mathrm{S}$ ratio 0.3$), 47 \%$ carbohydrate, and $16 \%$ protein, with $200 \mathrm{mg}$ of cholesterol per 1,000 cal. All subjects gave informed consent, and the study protocol was approved by the Clinical Research Subpanel of the National Heart, Lung, and Blood Institute.

ApoA-IV phenotyping. Phenotyping of apoA-IV was performed on whole plasma as previously described $(31,40)$. Briefly, plasma was delipidated according to Menzel et al. (28) and run on an isoelectric focusing (IEF) gel, using a pH range of 5-6 (Pharmalyte; Pharmacia LKB Biotechnology, Uppsala, Sweden). Proteins were electrotransferred to polyvinylidene difluoride membranes (Immobilon-P; Millipore Corp., Bedford, MA). After blocking with $3 \%$ bovine serum albumin in PBS, membranes were incubated for $1 \mathrm{~h}$ at room temperature with a 1:1,000 dilution of a polyclonal rabbit anti-human apoA-IV antibody raised in our laboratory. Membranes were washed twice with PBS for $10 \mathrm{~min}$, then incubated either with a goat anti-rabbit IgG antibody (Vectastain ABC kit; Vector Laboratories, Burlingame, CA) using 4-chloro-1-naphthol as substrate, or with a gold-labeled goat anti-IgG (Auroprobe; Amersham, Arlington Heights, IL) with silver enhancement, according to the directions of the manufacturers.

Isolation and iodination of apoA-IV-1, apoA-IV-2, and apoA-I. For isolation of the apoA-IV isoproteins, apoA-IV-1/1 and apoA-IV-2/2 homozygotes were identified by isoelectric focusing and immunoblotting as described above. Using PCR amplification of genomic DNA followed by DNA sequence analysis as previously described $(31,40)$, the apoA-IV-2/2 subject was confirmed to be homozygous for the $G$ to $T$ substitution at codon 360 , resulting in the substitution of histidine for glutamine at this residue.

ApoA-IV-1 and apoA-IV-2 were isolated from fasting lipoproteindepleted serum as previously described (41). Briefly, serum was adjusted to a density of $1.25 \mathrm{~g} / \mathrm{ml}$ with $\mathrm{KBr}$ and centrifuged at $330,000 \mathrm{~g}$ for $48 \mathrm{~h}$ in a $60 \mathrm{It}$ rotor (Beckman Instruments, Inc., Fullerton, CA). Lipoprotein-depleted serum was obtained by tube-slicing and extensively dialyzed against a buffer containing $0.15 \mathrm{M} \mathrm{NaCl}, 0.05 \mathrm{M} \mathrm{KPO}_{4}$, and $0.05 \%$ EDTA, pH 7.4. Samples were then incubated with a triglyceride-phospholipid emulsion (Intralipid), and lipid-protein complexes were recovered by a short centrifugation and delipidated with diethyl ether-ethanol ( $1: 1, \mathrm{vol} / \mathrm{vol})$. Delipidated proteins were solubilized in $10 \mathrm{mM}$ Tris- $\mathrm{HCl}, \mathrm{pH} \mathrm{8.2,} \mathrm{containing} 7 \mathrm{M}$ deionized urea and filtered through a $0.22 \mu \mathrm{m}$ membrane filter (Millipore Corp.). The protein solution was applied to a Mono Q HR 10/10 column equilibrated with the same Tris / urea buffer and run on a fast performance liquid chromatography (FPLC) system (Pharmacia LKB Biotechnology) at a flow rate of $2.0 \mathrm{ml} / \mathrm{min}$. The column was eluted for $40 \mathrm{~min}$ with a linear gradient of 0 to $200 \mathrm{mM} \mathrm{NaCl}$. Fractions containing apoA-IV were dialyzed immediately against $5 \mathrm{mM} \mathrm{NH}_{4} \mathrm{HCO}_{3}$, frozen, lyophylized, and stored at $-20^{\circ} \mathrm{C}$. Purity and identity of the isolated apoA-IV preparations were established by SDS gel electrophoresis and IEF, followed by Coomassie staining and immunoblotting with antibodies specific for apoA-IV as described above. Amino acid analysis was consistent with the known amino acid composition of apoA-IV $(5,42)$.
ApoA-I was isolated from the HDL of a healthy subject with normal lipid values by gel permeation and ion exchange chromatography (43).

Purified apoA-IV-1, apoA-IV-2, and apoA-I were iodinated by a modification of the iodine monochloride method as previously described (43). Briefly, lyophilized apolipoproteins were dissolved in a 6 $\mathrm{M}$ guanidine- $\mathrm{HCl}, 1 \mathrm{M}$ glycine buffer, $\mathrm{pH} 8.5$, then $5 \mathrm{mCi}$ of $\mathrm{Na}^{131} \mathrm{I}$ or $\mathrm{Na}^{125}$ I were added, followed by the iodine monochloride. Approximately $0.5 \mathrm{M}$ iodine was incorporated per mole protein. Radiolabeled apolipoproteins were reassociated immediately with separate aliquots of fresh fasting autologous plasma, and samples were extensively dialyzed against sterile PBS. Human serum albumin was added to a final concentration of $10 \mathrm{mg} / \mathrm{ml}$. The iodinated preparations were passed through a $0.22-\mu \mathrm{m}$ filter (Millipore Corp.) and tested for pyrogenicity and sterility. All samples were pyrogen-free, sterile, and contained no free iodine by radiochromatography. Radiolabeled apoA-IV-1, apoAIV-2, and apoA-I migrated to the expected positions on both SDS gel electrophoresis and IEF.

Study protocol. 10 subjects received radiolabeled apoA-IV-1 and apoA-IV-2 simultaneously, in studies that represented five separate iodinations of these apolipoproteins. Five subjects received radiolabeled apoA-IV-1 and apoA-I, and three received radiolabeled apoA-IV2 and apoA-I. $1 \mathrm{~d}$ before injection, the subjects were given an oral potassium iodide solution $(900 \mathrm{mg} / \mathrm{d}$ in divided doses) that was continued throughout the study. After a 12-h fast, subjects were injected intravenously with autologous plasma containing the radiolabeled apolipoproteins. Blood samples were obtained at $10 \mathrm{~min}, 1,3,6,12,18,24$, and $36 \mathrm{~h}$, and on days 2, 3, 4, and 7. Blood samples were drawn into tubes containing EDTA at a final concentration of $0.01 \%$. Plasma was separated by low speed centrifugation $(2,300 \mathrm{rpm}$ for $30 \mathrm{~min})$ at $4^{\circ} \mathrm{C}$. Sodium azide and aprotinin were added to each plasma sample at a final concentration of $0.05 \%$ and $200 \mathrm{KIU} / \mathrm{ml}$, respectively. Urine was collected continuously throughout the study. Radioactivity in plasma and urine was quantitated in a gamma counter (Autogamma 5260; Packard Instrument Co., Downers Grove, IL).

Plasma decay curves were constructed by dividing the plasma radioactivity at each time point by the radioactivity in the 10 -min plasma sample. Multiexponential functions were fit to the plasma decay curves utilizing the SAAM30 program (44). Residence times (RT) were obtained from the areas under the plasma decay curves. The fractional catabolic rates (FCR) are the reciprocals of the residence times. Apolipoprotein production rates $(\mathrm{PR})$ were calculated utilizing the formula: $P R=($ plasma apolipoprotein concentration $) \times($ plasma volume $)$ $\times(F C R) /(w t[k g])$. Plasma volume was assumed to be $4 \%$ of total body wt. For apoA-IV-1 / 1 homozygotes the FCR's of apoA-IV-1 were used and for the apoA-IV-2/2 homozygote the FCR of apoA-IV-2 was used in the determination of the apoA-IV production rates. For the apoA-IV-2/ 1 heterozygotes the means of the apoA-IV-1 and apoA-IV2 FCR's were used.

Gel filtration. Lipoproteins were separated according to size using gel filtration chromatography from fasting plasma 10 min after injection in two study subjects who were injected with radiolabeled apoAIV-1 and apoA-I. $3 \mathrm{ml}$ of plasma were applied through a flow adaptor to a $1.5 \times 75 \mathrm{~cm}$ glass column packed with $6 \%$ agarose beads (Bio-Gel A-5m, 200-400 mesh; Bio-Rad Labs, Richmond, CA) using an elution buffer of $0.9 \% \mathrm{NaCl}, 0.01 \%$ EDTA and $0.01 \% \mathrm{NaN}_{3}, \mathrm{pH}$ 7.4. The flow rate was $6 \mathrm{ml} / \mathrm{h}$ and fractions of $1.5 \mathrm{ml}$ were collected over $24 \mathrm{~h}$ at $4^{\circ} \mathrm{C}$. The column was calibrated with ultracentrifugally prepared VLDL ( $d$ $<1.006 \mathrm{~g} / \mathrm{ml})$ and HDL $(1.063-1.210 \mathrm{~g} / \mathrm{ml})$, as well as with BSA.

Immunoaffinity isolation of lipoproteins. ApoA-I-containing lipoproteins with apoA-II (LpA-I:A-II) and without apoA-II (LpA-I) were isolated from plasma $10 \mathrm{~min}$ after injection as previously described (43) in eight study subjects injected with both apoA-IV isoproteins. Briefly, monoclonal antibodies against apoA-I and apoA-II (45) were coupled to CNBr-activated Sepharose 4B (Pharmacia LKB Biotechnology). Plasma was first applied to the anti-apoA-II immunosorbent and apoA-II-containing particles were eluted with $3 \mathrm{M} \mathrm{NaSCN}$. The nonretained fraction from the anti-apoA-II column was then applied to an 
anti-apoA-I column and the retained apoA-I-containing particles were eluted with $3 \mathrm{M} \mathrm{NaSCN}$. All column samples were counted for apoAIV-associated radioactivity in order to determine the distribution of the radiolabeled apoA-IV in apoA-I-containing lipoproteins.

Ultracentrifugation. Lipoprotein density fractions (VLDL, LDL, HDL, VHDL) were isolated from $5 \mathrm{ml}$ plasma by sequential ultracentrifugation (46) and tube slicing. Radioactivity in the supranatant and infranatant fractions was determined after each centrifugation, and the ratio of radioactivity in the top/(top + bottom) was used to determine the total radioactivity in each density fraction at each time point.

Analytical methods. Plasma apoA-IV was quantitated by a noncompetitive ELISA similar to that previously described (47). Briefly, rabbit polyclonal antiserum to isolated human apoA-IV was purified using CM Affi-Gel Blue (Bio-Rad Labs), followed by ammonium sulfate precipitation. Microtiter plates were coated with anti-apoA-IV antibodies at a concentration of $1 \mu \mathrm{g} /$ well. After blocking with $0.8 \%$ casein, plasma samples diluted 1:2,000 were added to the wells and incubated at $4^{\circ} \mathrm{C}$ overnight. After washing, the same purified anti-apoA-IV antibodies conjugated with horseradish peroxidase (HRP) and diluted 1:2,000 were added to the wells and incubated at room temperature for $1.5 \mathrm{~h}$. After washing, HRP substrate was added, the reaction stopped in $20 \mathrm{~min}$, and absorbance read at $450 \mathrm{~nm}$ by an automated plate reader (Bio-Tek Instruments, Inc., Winooski, VT). A secondary plasma standard was developed after multiple measurements using a validated apoA-IV electroimmunoassay, which was previously described (48). Plasma cholesterol and triglycerides were quantitated by automated enzyme techniques on a VPSS analyzer (Abbott Labs, North Chicago, IL). HDL cholesterol was determined in plasma after dextran sulfate precipitation (49). Plasma apoA-I and apoA-II were quantitated by immunoturbidometric assay (Boehringer-Mannheim, Mannheim, Germany).

Statistical comparisons of apoA-IV-1 and apoA-IV-2 plasma kinetic parameters, fractional rate constants, intercepts, and density distributions were made using the paired Student's $t$ test. Linear regression analysis was performed using the least squares method. Means are expressed as \pm SEM.

\section{Results}

The clinical characteristics of the study subjects are presented in Table I. Values represent the mean of five measurements made during the kinetic study. Their weights and plasma lipid levels remained in steady state throughout the study. There were no significant differences between apoA-IV-1/1 homozygotes and apoA-IV-2/1 heterozygotes in any of the clinical parameters.

The total plasma kinetic parameters of apoA-IV metabolism are displayed in Table II. The mean apoA-IV-1 FCR was $2.42 \pm 0.11 \mathrm{~d}^{-1}$. The mean apoA-IV absolute production (or transport ) rate was $16.3 \pm 1.4 \mathrm{mg} / \mathrm{kg} / \mathrm{d}$. Linear regression analysis revealed that plasma apoA-IV concentrations were highly correlated with apoA-IV production rate $(r=0.84, P<0.001)$ and not correlated with apoA-IV FCR $(r=0.25, P=\mathrm{NS})$ (Fig. 1).

The mean FCR of the apoA-IV-2 isoprotein was $2.21 \pm 0.10$ $\mathrm{d}^{-1}$. Representative plasma curves of apoA-IV-1 and apoA-IV2 from two apoA-IV-2/1 heterozygotes are shown in Fig. 2. The FCR of apoA-IV- 2 was an average of $14 \pm 3 \%$ slower than that of apoA-IV-1 in the ten subjects who received both labeled apolipoproteins, significantly different by paired $t$ test $(P$ $=0.003)$. There were no significant differences in apoA-IV metabolism between the apoA-IV-1/1 homozygotes and the apoA-IV-2 / 1 heterozygotes. However, the FCR of apoA-IV-2 in the apoA-IV-2/2 homozygote was only $1.49 \mathrm{~d}^{-1}$, substantially slower than the mean apoA-IV-1 and apoA-IV-2 FCR's
Table I. Characterization of Study Subjects

\begin{tabular}{ccccccc}
\hline Subject & Sex & Age & BMI & \multicolumn{1}{c}{ TG } & \multicolumn{1}{c}{ TC } & HDL-C \\
\hline & & $y r$ & $k g / m^{2}$ & & $m g / d l$ & \\
1 & M & 19 & 30.5 & 110 & 228 & 35 \\
2 & M & 24 & 20.5 & 41 & 128 & 52 \\
3 & M & 20 & 23.0 & 195 & 133 & 44 \\
4 & M & 22 & 23.3 & 102 & 84 & 29 \\
5 & M & 22 & 22.4 & 80 & 181 & 42 \\
6 & F & 20 & 18.3 & 57 & 147 & 44 \\
7 & M & 22 & 21.4 & 88 & 161 & 47 \\
8 & F & 19 & 17.8 & 65 & 104 & 39 \\
9 & M & 21 & 19.6 & 131 & 150 & 30 \\
10 & F & 20 & 21.1 & 49 & 149 & 59 \\
11 & M & 22 & 26.9 & 82 & 178 & 49 \\
12 & M & 20 & 22.0 & 54 & 132 & 47 \\
13 & F & 20 & 22.4 & 84 & 185 & 44 \\
14 & M & 21 & 28.2 & 169 & 215 & 40 \\
15 & F & 21 & 22.5 & 69 & 147 & 37 \\
16 & F & 20 & 22.1 & 40 & 127 & 38 \\
17 & F & 21 & 19.7 & 62 & 146 & 50 \\
18 & M & 25 & 21.8 & 124 & 223 & 32 \\
& & 21 & 22.4 & 89 & 157 & 42 \\
Mean & & 0.4 & 0.8 & 10 & 9 & 2 \\
SEM & & & & & & \\
\hline & & & & & &
\end{tabular}

Table II. Kinetic Parameters of ApoA-IV-I and ApoA-IV-2 Metabolism

\begin{tabular}{cccccc}
\hline Subject & $\begin{array}{c}\text { ApoA-IV } \\
\text { Phenotype }\end{array}$ & $\begin{array}{c}\text { ApoA-IV } \\
\text { Conc }\end{array}$ & $\begin{array}{c}\text { ApoA-IV-1 } \\
\text { FCR }\end{array}$ & $\begin{array}{c}\text { ApoA-IV-2 } \\
\text { FCR }\end{array}$ & $\begin{array}{c}\text { ApoA-IV } \\
\text { PR }\end{array}$ \\
\hline & & $m g / d l$ & $d^{-1}$ & $d^{-1}$ & $m g / k g-d$ \\
1 & $1 / 1$ & 25.6 & 2.69 & 2.01 & 27.5 \\
2 & $1 / 1$ & 17.0 & 2.93 & 2.14 & 19.9 \\
3 & $1 / 1$ & 16.0 & 3.05 & 2.70 & 19.5 \\
4 & $1 / 1$ & 26.0 & 2.15 & 1.88 & 22.4 \\
5 & $1 / 1$ & 12.4 & 2.24 & - & 11.1 \\
6 & $1 / 1$ & 15.9 & 2.26 & - & 14.4 \\
7 & $1 / 1$ & 16.0 & 1.86 & - & 11.9 \\
8 & $1 / 1$ & 8.3 & 1.49 & - & 5.0 \\
9 & $1 / 1$ & 20.2 & 2.32 & - & 18.7 \\
10 & $2 / 1$ & 19.6 & 2.44 & 2.01 & 17.4 \\
11 & $2 / 1$ & 13.5 & 2.45 & 2.10 & 12.3 \\
12 & $2 / 1$ & 19.3 & 2.82 & 2.53 & 20.7 \\
13 & $2 / 1$ & 15.2 & 2.87 & 2.81 & 17.3 \\
14 & $2 / 1$ & 17.5 & 2.65 & 2.63 & 18.5 \\
15 & $2 / 1$ & 15.4 & 2.03 & 1.97 & 12.3 \\
16 & $2 / 1$ & 10.8 & - & 2.37 & - \\
17 & $2 / 1$ & 16.7 & - & 2.14 & - \\
18 & $2 / 2$ & 19.0 & - & 1.49 & 11.3 \\
& & 16.9 & $2.42^{*}$ & $2.21^{*}$ & 16.3 \\
Mean & & 1.1 & 0.11 & 0.10 & 1.4 \\
SEM & & & & & \\
& & & & & \\
\hline
\end{tabular}

Conc, concentration. FCR, fractional catabolic rate. PR, production rate. ${ }^{*}$ Difference significant by paired $t$ test, $P=0.003$. 


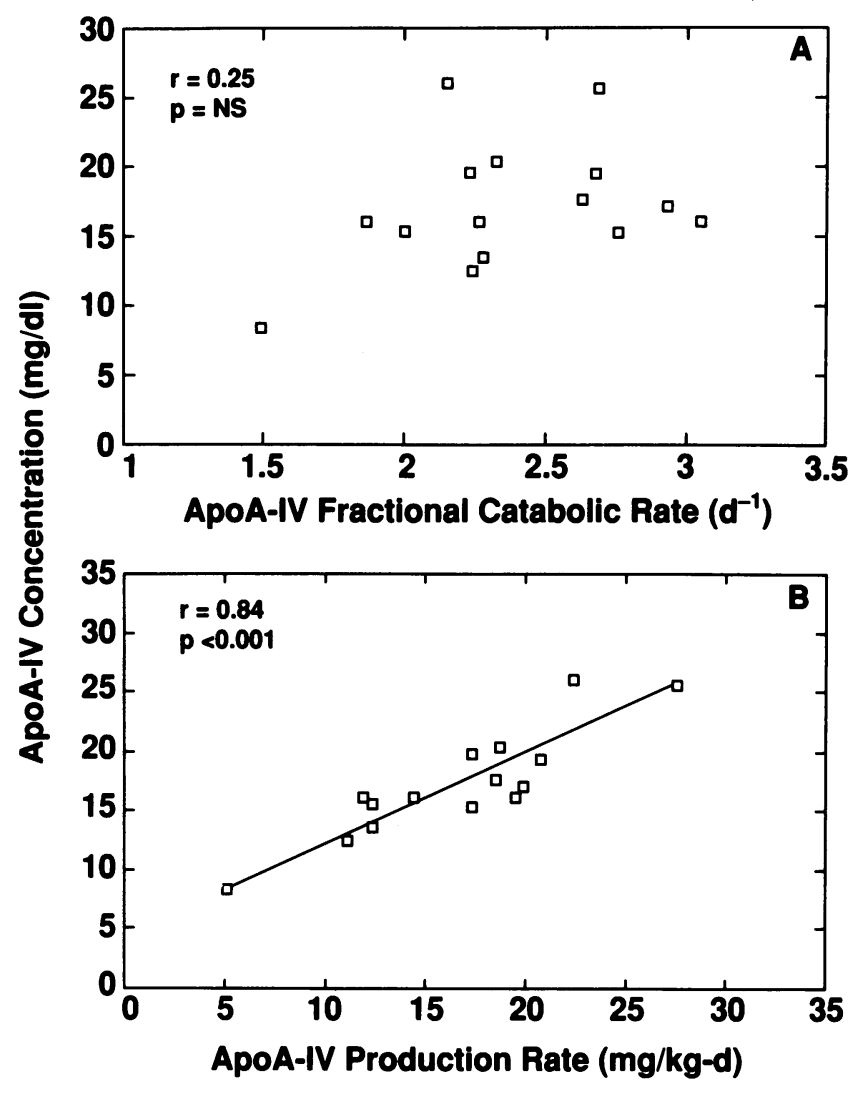

Figure 1. $(A)$ Correlation of apoA-IV fractional catabolic rates with plasma apoA-IV concentrations. $(B)$ Correlation of apoA-IV production rates with plasma apoA-IV concentrations.

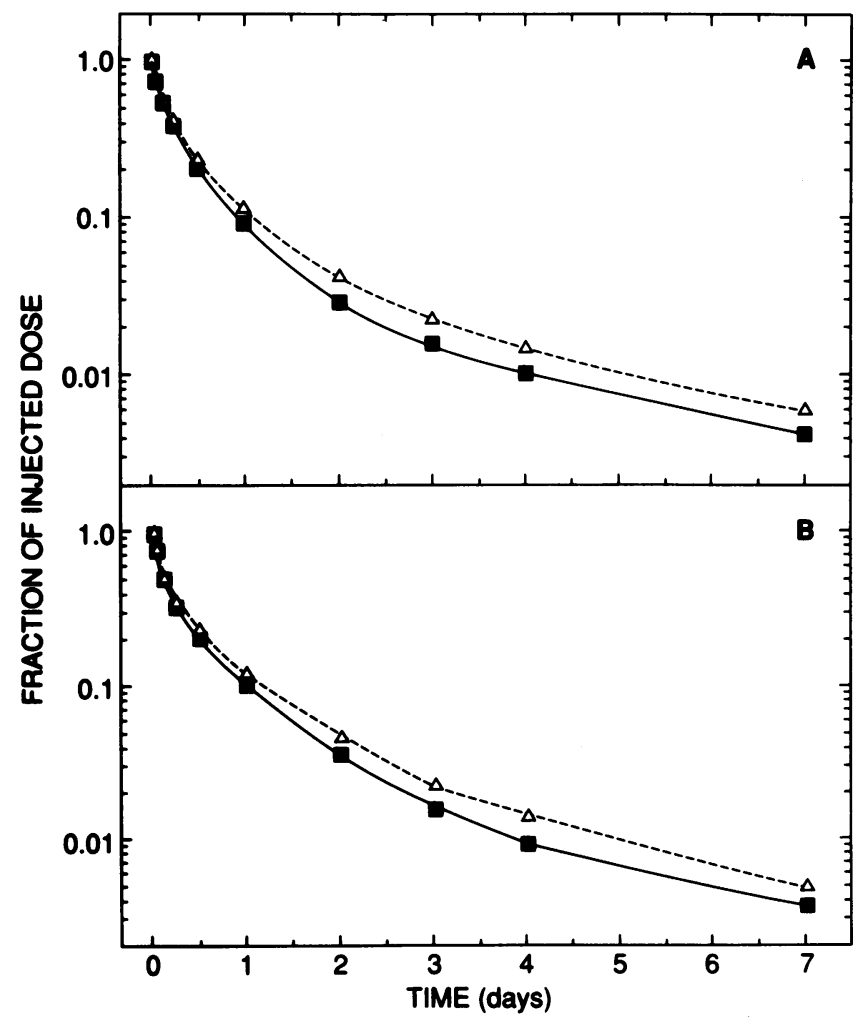

Figure 2. Total plasma radioactivity curves in two representative apoA-IV-2/1 heterozygotes. The squares and solid lines represent apoA-IV-1; the triangles and broken lines represent apoA-IV-2. in the other study subjects. In Fig. $3 A$ are shown the apoA-IV-2 plasma curves in the apoA-IV-2/ 2 homozygote, an apoA-IV$2 / 1$ heterozygote, and an apoA-IV-1/1 homozygote who were injected simultaneously with the same radiolabeled apoA-IV-2.

The difference in metabolism between apoA-IV-1 and apoA-IV-2 was not simply due to differential extravascular distribution, as illustrated by the representative urine/plasma $(\mathrm{U} / \mathrm{P})$ radioactivity ratios in Fig. 4 . The $\mathrm{U} / \mathrm{P}$ ratios for apoAIV-2 were consistently lower than those for apoA-IV-1 in the same subject, indicating a slower rate of degradation of apoAIV-2. The slower catabolism of apoA-IV-2 in the apoA-IV-2/2 homozygote can also be seen by the much lower apoA-IV-2/ 2 $\mathrm{U} / \mathrm{P}$ ratios in this subject compared with two subjects studied at the same time (Fig. $3 B$ ). By $4 \mathrm{~d}$ the U/P ratios of apoA-IV-1 and apoA-IV-2 were relatively equal, indicating that a slowly turning-over apoA-IV pool is catabolized at a similar rate for both forms of apoA-IV.

The heterogeneity of the U/P ratios over time indicates that there are distinct metabolic pools of apoA-IV with substantially different catabolic rates. The difference between apoA-IV-1 and apoA-IV-2 metabolism was further analyzed using the exponentials of the plasma decay curves. The apoAIV plasma decay curves were each fit to three different exponentials, with fractional rate constants $\mathrm{L} 1, \mathrm{~L} 2$, and $\mathrm{L} 3$, and intercepts $\alpha 1, \alpha 2$, and $\alpha 3$ (Table III). The kinetic difference between apoA-IV-1 and apoA-IV-2 was not due to differences in the fractional rate constants of the exponentials, but rather to significant differences in the intercepts, which represent the apparent distribution of the radiolabeled apolipoproteins among the three components as a fraction of the injected dose.

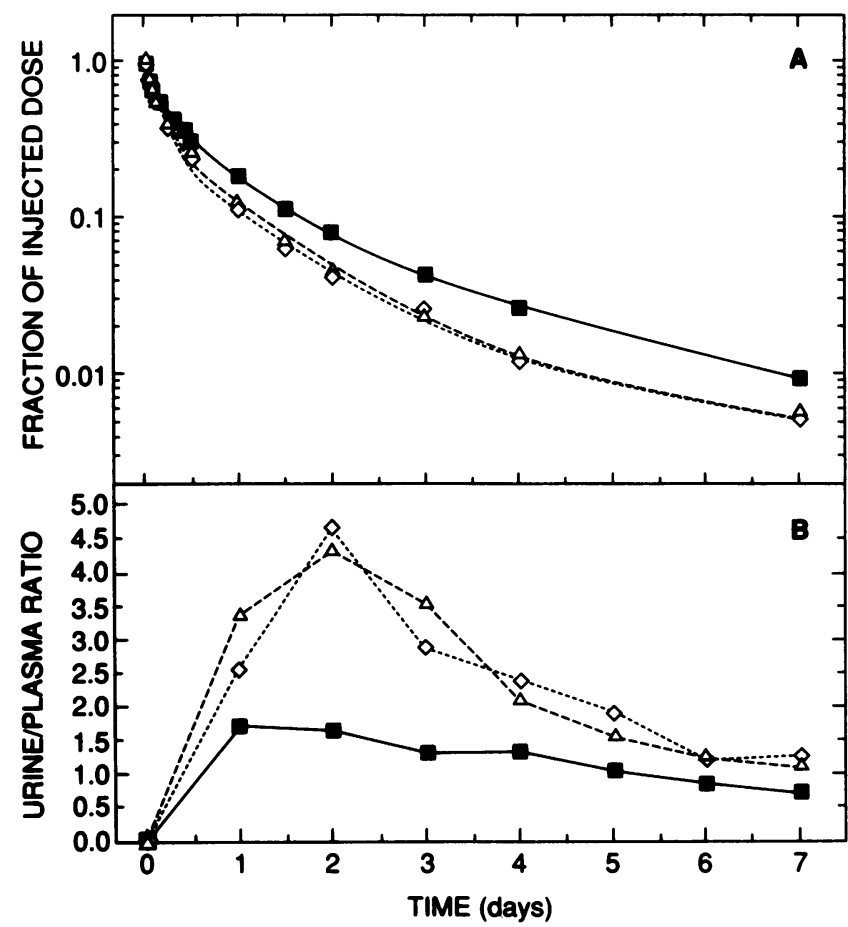

Figure 3. $(A)$ Total plasma radioactivity curves of apoA-IV-2 in an apoA-IV-2/2 homozygote (squares and solid line), an apoA-IV-2/1 heterozygote (triangles and solid line), and an apoA-IV-1 / 1 homozygote (diamonds and broken line). (B) Urine/plasma radioactivity ratio curves in the same three subjects. 


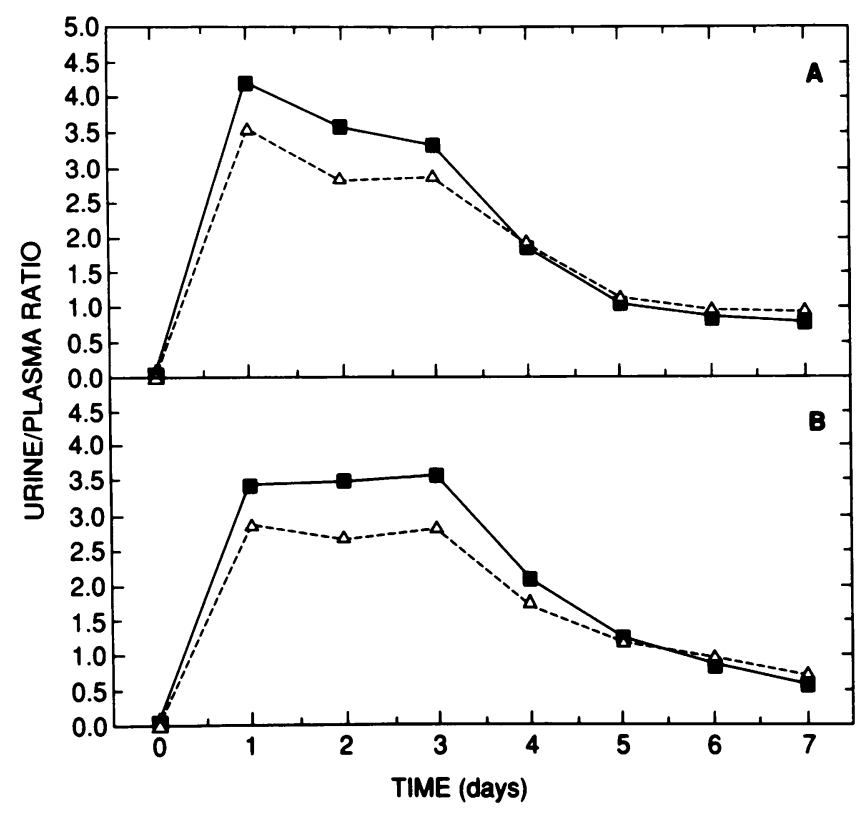

Figure 4. Urine/plasma radioactivity ratio curves in two representative apoA-IV-2/ 1 heterozygotes. The squares and solid lines represent apoA-IV-1; the triangles and broken lines represent apoA-IV-2.

Compared with apoA-IV-1, less apoA-IV-2 was associated with the most rapidly turning over component ( $\mathrm{Ll}$ ) and more with the more slowly turning over components (L2 and L3).

The distribution of apoA-IV according to lipoprotein particle size was determined by gel filtration chromatography 10 min after injection into two subjects who were injected with radiolabeled apoA-IV-1 and apoA-I (Fig. 5 ). By this chromatographic procedure, an average of $83 \%$ of the ${ }^{125} \mathrm{I}$-apoA-IV-1 and $95 \%$ of the apoA-IV mass was found to be associated with lipoprotein particles in the HDL and VHDL size range. There were two peaks of apoA-IV-associated radioactivity in this region: one coeluted with the HDL standard and the radiolabeled apoA-I peak and represented $29 \%$ of the total apoA-IV radioactivity; the other eluted between the HDL and BSA standards and represented $54 \%$ of the total apoA-IV radioactivity. There was some radiolabeled apoA-IV in the triglyceride-rich lipoprotein (TRL) fraction but virtually none in the lipid-deficient fraction (LDF).

Table III. Rate Constants (L) and Intercepts ( $\alpha)$ of the Three Exponentials of apo- $A-I V-1$ and apoA-IV-2 in the Ten Study Subjects Injected with both Radiolabeled Isoproteins ( \pm SEM)

\begin{tabular}{cccc}
\hline & ApoA-IV-1 & ApoA-IV-2 & $P^{*}$ \\
\hline Rate constants & \multicolumn{4}{c}{$d^{-1}$} \\
L1 & $8.83 \pm 0.53$ & $9.35 \pm 0.69$ & 0.28 \\
L2 & $1.67 \pm 0.11$ & $1.64 \pm 0.14$ & 0.48 \\
L3 & $0.34 \pm 0.06$ & $0.36 \pm 0.05$ & 0.21 \\
Intercepts & \multicolumn{2}{c}{ (fraction of injected dose) } \\
$\alpha 1$ & $0.613 \pm 0.028$ & $0.553 \pm 0.029$ & 0.0003 \\
$\alpha 2$ & $0.353 \pm 0.022$ & $0.394 \pm 0.021$ & 0.0004 \\
$\alpha 3$ & $0.034 \pm 0.008$ & $0.053 \pm 0.011$ & 0.003 \\
& & & \\
\hline
\end{tabular}

* Paired $t$ test.

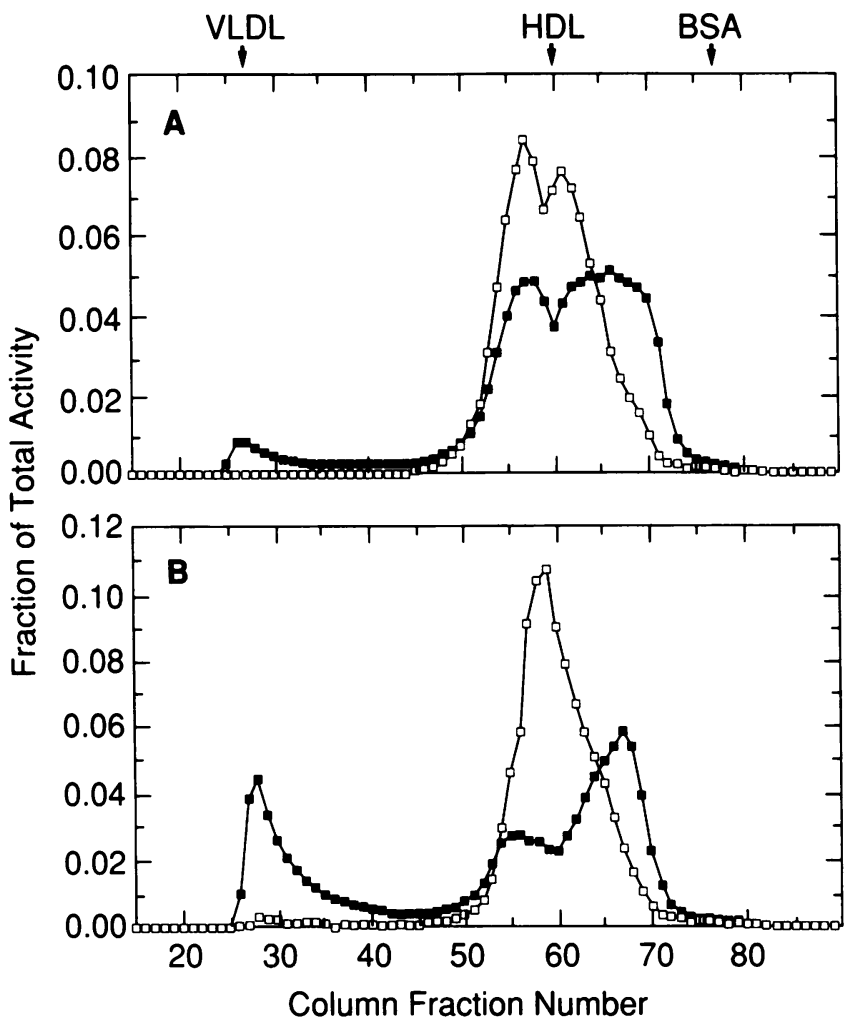

Figure 5. Distribution of radiolabeled ${ }^{125} \mathrm{I}$-apoA-IV-1 (closed squares) and ${ }^{131} \mathrm{I}$-apoA-I (open squares) after gel filtration chromatography of plasma drawn $10 \mathrm{~min}$ after injection in subject $6(A)$ and subject $5(B)$. Arrows mark the elution positions of the VLDL, HDL, and BSA standards. The $Y$-axis represents the fraction of total radioactivity recovered from the column.

The association of radiolabeled apoA-IV-1 and apoA-IV-2 with apoA-I-containing lipoproteins was determined in eight subjects by immunoaffinity chromatography of fasting plasma drawn 10 min after injection (Table IV). The majority of the apoA-I-associated apoA-IV was found in association with LpA-I:A-II rather than with LpA-I. A total of $71 \pm 6 \%$ of the apoA-IV- 1 and $66 \pm 4 \%$ of the apoA-IV- 2 were associated with apoA-I. The differences between apoA-IV-1 and apoA-IV-2 were not statistically significant.

The distribution of radiolabeled apoA-IV-1 and apoA-IV-2 was also determined after sequential ultracentrifugation of plasma from the 10 study subjects who were injected with both

Table IV. Distribution of Radiolabeled apoA-IV-1 and apoA-IV-2 in Lipoprotein particles LpA-I and LpA-I:A-II $10 \mathrm{~min}$ after Injection in Eight Study Subjects Injected with Both Radiolabeled Isoproteins ( \pm SEM)

\begin{tabular}{lccc}
\hline & ApoA-IV-1 & ApoA-IV-2 & $P *$ \\
\hline & & $\%$ & \\
LpA-I & $13 \pm 3$ & $16 \pm 4$ & NS \\
LpA-I:A-II & $58 \pm 5$ & $50 \pm 2$ & NS \\
$\begin{array}{l}\text { Total apoA-I-associated } \\
\text { (LpA-I + LpA-I:A-II) }\end{array}$ & $71 \pm 6$ & $66 \pm 4$ & NS \\
\hline
\end{tabular}

* Paired $t$ test. 
Table V. Distribution of Radiolabeled apoA-IV-1 and apoA-IV in Lipoprotein Density Subfractions 10 min after Injection in Ten Study Subjects Injected with Both Radiolabeled Isoproteins ( \pm SEM)

\begin{tabular}{lrll}
\hline & ApoA-IV-1 & ApoA-IV-2 & \multicolumn{1}{c}{$P^{*}$} \\
\hline $\mathrm{HDL}_{2}$ & $8.4 \pm 1.1$ & $10.2 \pm 1.2$ & 0.0006 \\
$\mathrm{HDL}_{3}$ & $12.4 \pm 1.1$ & $13.1 \pm 1.2$ & 0.069 \\
VHDL & $26.3 \pm 3.4$ & $23.9 \pm 3.1$ & 0.025 \\
$d>1.25$ & $46.3 \pm 2.9$ & $44.8 \pm 2.9$ & 0.163 \\
& & & \\
\hline
\end{tabular}

* Paired $t$ test.

radiolabeled isoproteins (Table $\mathrm{V}$ ). $10 \mathrm{~min}$ after injection, $<5 \%$ of the radioactivity was found in the $d<1.063 \mathrm{~g} / \mathrm{ml}$ (VLDL + LDL) fraction, but a highly reproducible fraction of the apoA-IV-associated radioactivity was found in the traditional HDL density range $(d=1.063-1.21 \mathrm{~g} / \mathrm{ml})$. There was a small but significant difference in the distribution of the two radiolabeled apoA-IV isoproteins: $20.8 \pm 1.3 \%$ of apoA-IV-1 was found in the HDL range, whereas $23.4 \pm 1.0 \%$ of apoA-IV-2 was HDL-associated ( $P=0.001$ by paired $t$ test). Conversely, in the VHDL range $(d=1.21-1.25 \mathrm{~g} / \mathrm{ml})$ there was more apoA-IV-1 than apoA-IV-2. In Fig. 6 are displayed the mean decay curves for HDL-associated $(d=1.063-1.21 \mathrm{~g} / \mathrm{ml})$ and non-HDL-associated (total counts minus HDL counts) apoAIV. In all subjects, the HDL-associated apoA-IV was catabolized significantly more slowly than the non-HDL-associated $(P<0.005$ for every time point after $3 \mathrm{~h}$ for both apoA-IV isoproteins).

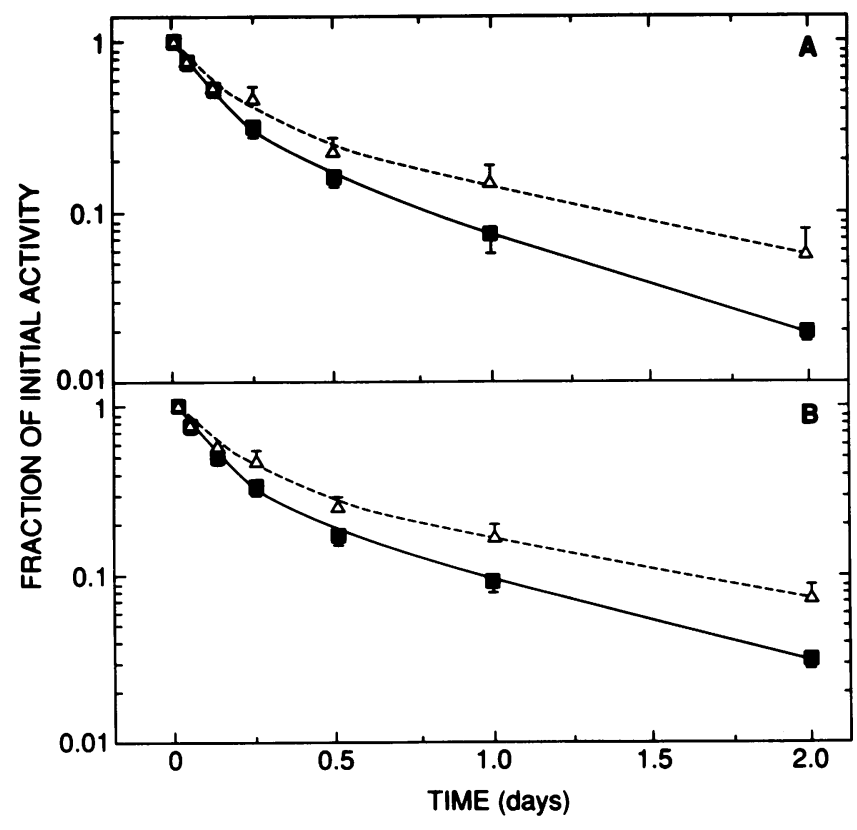

Figure 6. Plasma radioactivity curves for HDL-associated (triangles) and non-HDL-associated (squares) apoA-IV-1 $(A)$ and apoA-IV-2 (B). Data points represent mean values \pm SD.

\section{Discussion}

Despite considerable interest in apoA-IV as a potentially important participant in lipoprotein metabolism and reverse cholesterol transport, the plasma kinetics of apoA-IV in humans have not been extensively investigated. There have been three studies of apoA-IV metabolism in humans, but all involved the injection of radiolabeled apoA-IV which had first been reassociated with a lipoprotein density fraction prior to injection in a limited number of subjects. Ohta et al. (37) performed in vivo studies of radiolabeled human lymph apoA-IV reassociated with lymph TRL, plasma TRL, HDL, or the lipoprotein-deficient fraction (LDF) in five individuals, only one of whom was normolipidemic. Studies were carried out for only $2 \mathrm{~d}$ and no kinetic parameters were reported for the injected apoA-IV. Ghiselli et al. (38) reported the kinetics of radiolabeled human lymph apoA-IV reassociated with TRL, HDL, or LDF in six normolipidemic subjects and concluded that the catabolism of apoA-IV within the LDF was faster than that in HDL. Recently, Malmendier et al. (39) reported studies of human serum apoA-IV reassociated with HDL prior to injection in five normolipidemic subjects and found that the average fractional catabolic rate of HDL-associated apoA-IV was only 0.7 $\mathrm{d}^{-1}$. All of these studies were performed without knowledge of the apoA-IV phenotype of the source of the purified apoA-IV or of the study subjects. In the current investigation, we established the plasma kinetics of total apoA-IV and directly compared the metabolism of the two major isoforms of apoA-IV in normolipidemic subjects of defined apoA-IV phenotype.

ApoA-IV-1, the major apoA-IV isoprotein, was found to have a mean FCR of $2.42 \mathrm{~d}^{-1}$, making it one of the most rapidly catabolized apolipoproteins in humans. This rapid turnover is supported by recent data from our laboratory (Verges, B., et al., unpublished data) using endogenous labeling of apoA-IV with stable isotopically labeled amino acids. The mean apoA-IV absolute production, or transport, rate was 16.3 $\mathrm{mg} / \mathrm{kg}$ per d, which is in a range similar to that of apoA-I ( 50 , 51 ). It has been previously demonstrated that apoA-IV is similar in structure to apoA-I, has excellent access to the interstitial space, promotes cholesterol efflux from cells, activates LCAT, associates with HDL, may modulate CETP activity, and binds specifically to hepatic tissue. The high rate of in vivo apoA-IV transport lends further support to the hypothesis that despite its low steady state concentration relative to apoA-I, apoA-IV may play a role in reverse cholesterol transport in humans.

Plasma concentrations of apoA-I are more highly correlated with apoA-I fractional catabolic rate than with the rate of apoA-I production $(50,52,53)$. In contrast, we found that the major determinant of plasma apoA-IV concentrations was the apoA-IV production rate in these normolipidemic subjects, with no correlation between apoA-IV level and catabolic rate. Although plasma apoA-IV levels have been shown to increase in response to increased dietary fat intake (54), all subjects in our studies were on a controlled metabolic diet. Hence, the variation in apoA-IV production rate among individuals suggests that nondietary factors, possibly genetic, determine the rate of intestinal apoA-IV synthesis. In several strains of mice, differences in cis-acting elements near the apoA-IV gene have been shown to affect apoA-IV gene expression (55). Our results suggest that similar genetic variation in apoA-IV gene 
expression may exist in humans and may be an important determinant of plasma apoA-IV levels.

The metabolism of apoA-IV is highly heterogeneous, as indicated by the $U / P$ ratio curves and the exponentials of the plasma decay curves, suggestive of several kinetic compartments with very different turnover rates. The true distribution of apoA-IV in human plasma has not been established. Early reports indicated that after ultracentrifugation, most of the apoA-IV in fasting plasma was found in the $d>1.21$ density range $(4,56,57)$. However, Lagrost et al. (58) found $18 \%$ of apoA-IV in the $d<1.21 \mathrm{~g} / \mathrm{ml}$ density range (mostly HDL) after ultracentrifugation. Using traditional gel filtration chromatography, $\sim 20 \%$ of plasma apoA-IV has been reported to be associated with $\operatorname{HDL}(4,38,57,59,60)$. On the other hand, Lagrost et al. (58), using Superose 12-HR columns (Sigma Chemical Co., St. Louis, MO) on FPLC (which decreases separation time and may improve resolution), found about $70 \%$ of apoA-IV mass associated with HDL; they also found that $70 \%$ of apoA-IV immunoprecipitated with an antibody to apoA-I (58). Finally, when apoA-IV-containing particles were isolated from plasma by anti-apoA-IV immunoaffinity chromatography and separated by FPLC gel filtration, most of the apoA-IV was found in particles in the small HDL range (12).

In our studies, gel filtration chromatography indicated that the majority of the radiolabeled apoA-IV and apoA-IV mass were associated with lipoprotein particles of the HDL and VHDL size range. Immunoaffinity chromatography suggested that a substantial amount of labeled apoA-IV was associated with apoA-I; the majority of this apoA-I-associated apoA-IV was found to be with LpA-I:A-II rather than LpA-I, consistent with a previous report (39). A portion of radiolabeled apoA-IV (20-25\%) remained consistently associated with HDL even after sequential ultracentrifugation. The catabolic rate of the HDL-associated apoA-IV was substantially slower than that of non-HDL-associated apoA-IV and was similar to that of radiolabeled apoA-IV reassociated with HDL prior to injection (38, 39). Furthermore, the tails of the $U / P$ ratio curves had a mean $\mathrm{U} / \mathrm{P}$ ratio that approached the FCR of apoA-IV injected on HDL (39). Hence, our data is consistent with the existence of a pool of HDL-associated apoA-IV which turns over at slower rate than the remainder of plasma apoA-IV.

The discrepancies in the literature concerning the true distribution of apoA-IV in human plasma may have several possible explanations. First, apoA-IV has very labile binding to lipoproteins, especially HDL (23), and therefore its apparent distribution may be very sensitive to the exact methodology used to isolate the lipoprotein particles, as well as to factors like plasma storage conditions and LCAT and CETP activity. Second, there may be differences in immunoreactivity of apoA-IV on different lipoprotein particles that affect its immunoquantitation in lipoprotein subfractions and may vary among different assays. Finally, we found by both gel filtration and ultracentrifugation that a substantial amount of apoA-IV was present in the VHDL range, which has not traditionally been considered part of HDL but probably represents small dense lipoprotein particles rather than free protein (61).

The FCR of apoA-IV-2 was an average of $14 \pm 3 \%$ slower than that of apoA-IV-1 in the ten subjects who were injected with both radiotracers, a small but significant difference. Furthermore, the catabolism of apoA-IV-2 was $35 \%$ slower in an apoA-IV-2/2 homozygote than the mean apoA-IV-2 FCR in the other 12 subjects who were injected with this tracer. The $U / P$ ratio curves confirmed the metabolic difference between apoA-IV-1 and apoA-IV-2, as the U/P ratios for apoA-IV-2 were consistently lower than those for apoA-IV-1. Analysis of the individual plasma curve exponentials suggested that the major reason for the slower catabolism of apoA-IV-2 than apoA-IV-1 was a preferential distribution of apoA-IV-2 in more slowly turning-over compartments, rather than differences in the fractional rate constants themselves. This was supported by the finding that a higher fraction of apoA-IV-2 was found in association with HDL after sequential ultracentrifugation, and is consistent with the observation that apoA-IV-2 has more alpha-helical structure and higher affinity for phospholipids than does apoA-IV-1 (36). ApoA-IV can self-associate and is likely to exist at least in part as a homodimer under physiologic conditions (62), possibly in association with some lipid and even other apolipoproteins (61). A difference between the two isoproteins in rate of self-association could affect their distribution and influence their kinetic rates, as was previously proposed (62). The apoA-IV-2 isoprotein had an even slower catabolic rate in the apoA-IV-2/2 homozygote than in the apoA-IV-2/ 1 heterozygotes, possibly because in the homozygote all apoA-IV dimers are true homodimers of apoA-IV-2 rather than "mixed" apoA-IV-2/1 dimers. Further studies will be needed to explore these hypotheses.

In summary, apoA-IV has a rapid fractional catabolic rate ten times that of apoA-I and an absolute transport rate comparable to that of apoA-I. In contrast to apoA-I, plasma concentrations of apoA-IV in normolipidemic subjects are determined by the apoA-IV production rate. The catabolism of the common variant apoA-IV-2 isoprotein is slower than that of the wild-type apoA-IV-1, primarily because of an altered distribution in the plasma. This may be related to the apparent effects of the apoA-IV-2 allele on plasma lipid and apolipoprotein concentrations. These studies provide further insight into the complex nature of apoA-IV metabolism in humans.

\section{Acknowledgments}

We are indebted to Glenda Talley, Yoshiko Doherty, Rosemary Ronan, and Sabine Motzny for excellent technical assistance, Betty Kuzmik and the nursing staff of the 8 East inpatient ward of the $\mathrm{Na}$ tional Institutes of Health Clinical Center for care of the study subjects, Patti Riggs and the Metabolic Kitchen for invaluable dietary assistance, Loan Kusterbeck for secretarial assistance, and the study subjects for participating.

A. Steinmetz is the recipient of a grant from the Deutsche Forschungsgemeinschaft (Germany).

\section{References}

1. Weisgraber, K. H., T. P. Bersot, and R. W. Mahley. 1978. Isolation and characterization of an apoprotein from the $\mathrm{d}$ less than 1.006 lipoproteins of human and canine lymph homologous with the rat A-IV apoprotein. Biochem. Biophys. Res. Commun. 85:287-292.

2. Utermann, G., and U. Beisiegel. 1979. Apolipoprotein A-IV: a protein occurring in human mesenteric lymph chylomicrons and free in plasma. Isolation and quantification. Eur. J. Biochem. 99:333-343.

3. Green, P. H., R. M. Glickman, C. D. Saudek, C. B. Blum, and A. R. Tall. 1979. Human intestinal lipoproteins. Studies in chyluric subjects. J. Clin. Invest. $64: 233-242$. 
4. Green, P. H., R. M. Glickman, J. W. Riley, and E. Quinet. 1980. Human apolipoprotein A-IV. Intestinal origin and distribution in plasma. J. Clin. Invest. 65:911-919.

5. Weinberg, R. B., and A. M. Scanu. 1983. Isolation and characterization of human apolipoprotein A-IV from lipoprotein-depleted serum. J. Lipid Res. 24:52-59.

6. Wu, A. L., and H. G. Windmueller. 1979. Relative contributions by liver and intestine to individual plasma apolipoproteins in the rat. J. Biol. Chem. 254:7316-7322.

7. Karathanasis, S. K., I. Yunis, and V. I. Zannis. 1986. Structure, evolution, and tissue-specific synthesis of human apolipoprotein AIV. Biochemistry. 25:3962-3970.

8. Kraft, H. G., H. J. Menzel, F. Hoppichler, W. Vogel, and G. Utermann. 1989. Changes of genetic apolipoprotein phenotypes caused by liver transplantation. Implications for apolipoprotein synthesis. J. Clin. Invest. 83:137-142.

9. Karanthanasis, S. K., P. Oettgen, I. A. Haddad, and S. E. Antonarakis. 1986. Structure, evolution, and polymorphisms of the human apolipoprotein A4 gene (APOA4). Proc. Natl. Acad. Sci. USA. 83:8457-8461.

10. Sloop, C. H., L. Dory, B. R. Krause, C. Castle, and P. S. Roheim. 1983. Lipoproteins and apolipoproteins in peripheral lymph of normal and cholesterolfed dogs. Atherosclerosis. 49:9-21.

11. Duverger, N., N. Ghalim, N. Theret, P. Duchateau, G. Aguie, G. Ailhaud, G. Castro, and J. C. Fruchart. 1991. Lipoprotein A-I containing particles. $A d v$. Exp. Med. Biol. 285:93-99.

12. Duverger, N., N. Ghalim, G. Ailhaud, A. Steinmetz, J. C. Fruchart, and G. Castro. 1993. Characterization of apo A-IV-containing lipoprotein particles isolated from human plasma and interstitial fluid. Arterioscler. Thromb. 13:126132.

13. Goldberg, I. J., C. A. Scheraldi, L. K. Yacoub, U. Saxena, and C. L. Bisgaier. 1990. Lipoprotein ApoC-II activation of lipoprotein lipase. Modulation by apolipoprotein A-IV. J. Biol. Chem. 265:4266-4272.

14. Savion, N., and A. Gamliel. 1988. Binding of apolipoprotein A-I and apolipoprotein A-IV to cultured bovine aortic endothelial cells. Arteriosclerosis. 8:178-186.

15. Stein, O., Y. Stein, M. Lefevre, and P. S. Roheim. 1986. The role of apolipoprotein A-IV in reverse cholesterol transport studied with cultured cells and liposomes derived from an ether analog of phosphatidylcholine. Biochim. Biophys. Acta. 878:7-13.

16. Steinmetz, A., R. Barbaras, N. Ghalim, V. Clavey, J. C. Fruchart, and G. Ailhaud. 1990. Human apolipoprotein A-IV binds to apolipoprotein A-I/A-II receptor sites and promotes cholesterol efflux from adipose cells. J. Biol. Chem. 265:7859-7863.

17. Steinmetz, A., and G. Utermann. 1985. Activation of lecithin: cholesterol acyltransferase by human apolipoprotein A-IV. J. Biol. Chem. 260:2258-2264.

18. Chen, C. H., and J. J. Albers. 1985. Activation of lecithin: cholesterol acyltransferase by apolipoproteins E-2, E-3, and A-IV isolated from human plasma. Biochim. Biophys. Acta. 836:279-285.

19. Barter, P. J., O. V. Rajaram, L. B. Chang, K. A. Rye, P. Gambert, L. Lagrost, C. Ehnholm, and N. H. Fidge. 1988. Isolation of a high-density-lipoprotein conversion factor from human plasma. A possible role of apolipoprotein A-IV as its activator. Biochem. J. 254:179-184.

20. Lagrost, L., P. Gambert, V. Dangremont, A. Athias, and C. Lallemant. 1990. Role of cholesteryl ester transfer protein (CETP) in the HDL conversion process as evidenced by using anti-CETP monoclonal antibodies. J. Lipid Res. 31:1569-1575.

21. Dvorin, E., N. L. Gorder, D. M. Benson, and A. M. Gotto, Jr. 1986. Apolipoprotein A-IV. A determinant for binding and uptake of high density lipoproteins by rat hepatocytes. J. Biol. Chem. 261:15714-15718.

22. Weinberg, R. B., and C. S. Patton. 1990. Binding of human apolipoprotein A-IV to human hepatocellular plasma membranes. Biochim. Biophys. Acta. 1044:255-261.

23. Weinberg, R. B., J. A. Ibdah, and M. C. Phillips. 1992. Adsorption of apolipoprotein A-IV to phospholipid monolayers spread at the air/water interface. J. Biol. Chem. 267:8977-8983.

24. Utermann, G., G. Feussner, G. Franceschini, J. Haas, and A. Steinmetz. 1982. Genetic variants of group A apolipoproteins. Rapid methods for screening and characterization without ultracentrifugation. J. Biol. Chem. 257:501-507.

25. Menzel, H. J., P. M. Kovary, and G. Assmann. 1982. Apolipoprotein A-IV polymorphism in man. Hum. Genet. 62:349-352.

26. Lohse, P., and H. B. Brewer, Jr. 1991. Genetic polymorphism of apolipoprotein A-IV. Curr. Opin. Lipidol. 2:90-95.

27. Kamboh, M. I., and R. E. Ferrell. 1987. Genetic studies of human apolipoproteins. I. Polymorphism of apolipoprotein A-IV. Am. J. Hum. Genet. 41:119-127.

28. Menzel, H. J., E. Boerwinkle, S. Schrangl-Will, and G. Utermann. 1988 Human apolipoprotein A-IV polymorphism: frequency and effect on lipid and lipoprotein levels. Hum. Genet. 79:368-372.

29. de Knijff, P., M. Rosseneu, U. Beisiegel, W. de Keersgieter, R. R. Frants, and L. M. Havekes. 1988. Apolipoprotein A-IV polymorphism and its effect on plasma lipid and apolipoprotein concentrations. J. Lipid Res. 29:1621-1627.

30. Menzel, H. J., G. Sigurdsson, E. Boerwinkle, S. Schrangl-Will, H. Dieplinger, and G. Utermann. 1990. Frequency and effect of human apolipoprotein A-IV polymorphism on lipid and lipoprotein levels in an Icelandic population. Hum. Genet. 84:344-346.

31. Lohse, P., M. R. Kindt, D. J. Rader, and H. B. Brewer, Jr. 1990. Genetic polymorphism of human plasma apolipoprotein A-IV is due to nucleotide substitutions in the apolipoprotein A-IV gene. J. Biol. Chem. 265:10061-10064.

32. Boerwinkle, E., S. Visvikis, and L. Chan. 1991. Two polymorphisms for amino acid substitutions in the ApoA4 gene. Nucleic Acids Res. 18:4966.

33. Tenkanen, H., M. Lukka, M. Jauhiainen, J. Metso, M. Baumann, L. Peltonen, and C. Ehnholm. 1991. The mutation causing the common apolipoprotein A-IV polymorphism is a glutamine to histidine substitution of amino acid 360. Arterioscler. Throm. 11:851-856.

34. Eichner, J. E., L. H. Kuller, R. E. Ferrell, and M. I. Kamboh. 1989. Phenotypic effects of apolipoprotein structural variation on lipid profiles: II. Apolipoprotein A-IV and quantitative lipid measures in the healthy women study. Genet. Epidemiol. 6:493-499.

35. von Eckardstein, A., H. Funke, M. Schulte, M. Erren, H. Schulte, and G. Assmann. 1992. Nonsynonymous polymorphic sites in the apolipoprotein (apo) A-IV gene are associated with changes in the concentration of apo B- and apo A-I-containing lipoproteins in a normal population. Am. J. Hum. Genet. 50:1115-1128.

36. Weinberg, R. B., M. K. Jordan, and A. Steinmetz. 1990. Distinctive structure and function of human apolipoprotein variant ApoA-IV-2. J. Biol. Chem. 265:18372-18378.

37. Ohta, T., N. H. Fidge, and P. J. Nestel. 1985. Studies on the in vivo and in vitro distribution of apolipoprotein A-IV in human plasma and lymph. J. Clin. Invest. 76:1252-1260.

38. Ghiselli, G., S. Krishnan, Y. Beigel, and A. M. Gotto, Jr. 1986. Plasma metabolism of apolipoprotein A-IV in humans. J. Lipid Res. 27:813-827.

39. Malmendier, C. L., J.-F. Lontie, L. Lagrost, C. Delcroix, D. Y. Dubois, and P. Gambert. 1991. In vivo metabolism of apolipoproteins A-IV and A-I associated with high density lipoprotein in normolipidemic subjects. $J$. Lipid Res. 32:801-808.

40. Lohse, P., M. R. Kindt, D. J. Rader, and H. B. Brewer, Jr. 1990. Human plasma apolipoproteins A-IV-0 and A-IV-3. Molecular basis for two rare variants of apolipoprotein A-IV-1. J. Biol. Chem. 265:12734-12739.

41. Steinmetz, A., V. Clavey, N. Vu-Dac, H. Kaffarnik, and J. C. Fruchart. 1989. Purification of human apolipoprotein A-IV by fast protein liquid chromatography. J. Chromatogr. 487:154-160.

42. Elshourbagy, N. A., D. W. Walker, M. S. Boguski, J. I. Gordon, and J. M Taylor. 1986. The nucleotide and derived amino acid sequence of human apolipoprotein A-IV mRNA and the close linkage of its gene to the genes of apolipoproteins A-I and C-III. J. Biol. Chem. 261:1998-2002.

43. Rader, D. J., G. Castro, L. A. Zech, J. C. Fruchart, and H. B. Brewer, Jr. 1991. In vivo metabolism of apolipoprotein A-I on high density lipoprotein particles LpA-I and LpA-I, A-II. J. Lipid Res. 32:1849-1859.

44. Berman, M., and M. Weiss. 1978. SAAM Manual, DHEW Publ. No. (NIH) 78. National Institutes of Health, Bethesda, MD.

45. Betard, C., N. Vu-Dac, H. Mezdour, A. C. Nestruck, A. Leroy, and J. C. Fruchart. 1987. Standardization of an enzymometric assay for apolipoprotein A-I by using mixtures of monoclonal antibodies. J. Clin. Chem. Clin. Biochem. 25:893-899.

46. Havel, R. J., H. A. Eder, and J. H. Bragdon. 1955. The distribution and chemical composition of ultracentrifugally separated lipoproteins in human serum. J. Clin. Invest. 34:1345-1353.

47. Rosseneu, M., G. Michiels, W. de Keersgieter, J. Bury, J. P. De Slypere, H Dieplinger, and G. Utermann. 1988. Quantification of human apolipoprotein A-IV by "sandwich:-type enzyme-linked immunosorbent assay. Clin. Chem. 34:739-743.

48. Steinmetz, A., P. Czekelius, E. Thiemann, S. Motzny, and H. Kaffarnik. 1988. Changes of apolipoprotein A-IV in the human neonate: evidence for different inductions of apolipoproteins A-IV and A-I in the postpartum period. Atherosclerosis. 69:21-27.

49. Warnick, G. R., M. C. Cheung, and J. J. Albers. 1979. Comparison of current methods for high-density lipoprotein cholesterol quantitation. Clin. Chem. 25:596-604.

50. Schaefer, E. J., L. A. Zech, L. L. Jenkins, T. J. Bronzert, E. A. Rubalcaba, R. T. Lindgren, R. L. Aamodt, and H. B. Brewer, Jr. 1982. Human apolipoprotein A-I and A-II metabolism. J. Lipid Res. 23:850-862.

51. Brinton, E. A., S. Eisenberg, and J. L. Breslow. 1990. A low-fat diet decreases high density lipoprotein (HDL) cholesterol levels by decreasing HDL apolipoprotein transport rates. J. Clin. Invest. 85:144-151.

52. Brinton, E. A., S. Eisenberg, and J. L. Breslow. 1989. Elevated high density lipoprotein cholesterol levels correlate with decreased apolipoprotein A-I and A-II fractional catabolic rate in women. J. Clin. Invest. 84:262-269. 
53. Brinton, E. A., S. Eisenberg, and J. L. Breslow. 1991. Increased apo A-I and apo A-II fractional catabolic rate in patients with low high density lipoprotein-cholesterol levels with or without hypertriglyceridemia. J. Clin. Invest. 87:536-544.

54. Weinberg, R. B., C. Dantzker, and C. S. Patton. 1990. Sensitivity of serum apolipoprotein A-IV levels to changes in dietary fat content. Gastroenterology. 98:17-24.

55. Williams, S. C., S. G. Grant, K. Reue, B. Carrasquillo, A. J. Lusis, and A. J. Kinniburgh. 1989. cis-acting determinants of basal and lipid-regulated apolipoprotein A-IV expression in mice. J. Biol. Chem. 264:19009-19016.

56. Beisiegel, U., and G. Utermann. 1979. An apolipoprotein homolog of rat apolipoprotein A-IV in human plasma. Isolation and partial characterization. Eur. J. Biochem. 93:601-608.

57. Bisgaier, C. L., O. P. Sachdev, L. Megna, and R. M. Glickman. 1985. Distribution of apolipoprotein A-IV in human plasma. J. Lipid Res. 26:11-25.

58. Lagrost, L., P. Gambert, M. Boquillon, and C. Lallemant. 1989. Evidence for high density lipoproteins as the major apolipoprotein A-IV-containing fraction in normal human serum. J. Lipid Res. 30:1525-1534.

59. Bisgaier, C. L., O. P. Sachdev, E. S. Lee, K. J. Williams, C. B. Blum, and R. M. Glickman. 1987. Effect of lecithin:cholesterol acyltransferase on distribution of apolipoprotein A-IV among lipoproteins of human plasma. J. Lipid Res. 28:693-703.

60. Bisgaier, C. L., M. V. Siebenkas, C. B. Hesler, T. L. Swenson, C. B. Blum, Y. L. Marcel, R. W. Milne, R. M. Glickman, and A. R. Tall. 1989. Effect of a neutralizing monoclonal antibody to cholesteryl ester transfer protein on the redistribution of apolipoproteins A-IV and $\mathrm{E}$ among human lipoproteins. $J$. Lipid Res. 30:1025-1031.

61. Ohta, T., N. H. Fidge, and P. J. Nestel. 1984. Characterization of apolipoprotein A-IV complexes and A-IV isoforms in human lymph and plasma lipoproteins. J. Biol. Chem. 259:14888-14893.

62. Weinberg, R. B., and M. S. Spector. 1985. The self-association of human apolipoprotein A-IV. Evidence for an in vivo circulating dimeric form. J. Biol. Chem. 260:14279-14286. 